\title{
Sur le parallèle. Avant-propos
}

Johann Goeken et Catherine Schneider

\section{(2) OpenEdition}

Journals

Édition électronique

URL : https://journals.openedition.org/rhetorique/1108

DOI : 10.4000/rhetorique. 1108

ISSN : 2270-6909

\section{Éditeur}

UGA Éditions/Université Grenoble Alpes

\section{Édition imprimée}

ISBN : 978-2-37747-296-3

\section{Référence électronique}

Johann Goeken et Catherine Schneider, «Sur le parallèle. Avant-propos », Exercices de rhétorique [En ligne], 16 | 2021, mis en ligne le 12 mai 2021, consulté le 19 mai 2021. URL : http://

journals.openedition.org/rhetorique/1108; DOI : https://doi.org/10.4000/rhetorique.1108

Ce document a été généré automatiquement le 19 mai 2021.

\section{(c) (i) (2)}

Les contenus de la revue Exercices de rhétorique sont mis à disposition selon les termes de la Licence Creative Commons Attribution - Pas d'Utilisation Commerciale - Partage dans les Mêmes Conditions 4.0 International. 


\title{
Sur le parallèle. Avant-propos
}

\author{
Johann Goeken et Catherine Schneider
}

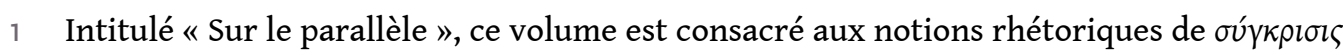
et comparatio - termes que l'on traduit habituellement par «comparaison » et que l'on rend aussi parfois par le concept plus englobant de "parallèle ». Illustrant plusieurs aspects de la question, ce florilège de contributions entend suggérer l'intérêt et le succès de ces notions depuis l'Antiquité grecque jusqu'à la modernité classique et audelà.

\section{Parallèle et comparaison}

2 Le mot "parallèle" sert à traduire le substantif grec бúүкрıбı, qui signifie aussi « comparaison » par le biais du latin comparatio. Quant à l'adjectif «parallèle », il vient

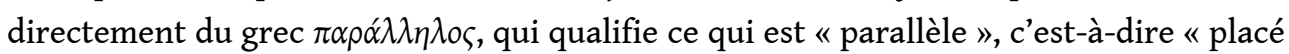
en regard ", et peut impliquer l'idée de similitude ou d'analogie.

3 En français, l'adjectif "parallèle» "se dit de lignes, de surfaces qui, en géométrie euclidienne, ne se rencontrent pas » (on parle ainsi de "droites parallèles », qui vont dans la même direction); il signifie en outre " qui suit la même direction ", "qui a lieu en même temps, porte sur le même objet» (par exemple: un marché, un cours parallèle); est encore « parallèle » ce « qui présente une comparaison suivie entre deux objets ", par exemple les Vies parallèles de Plutarque (on parle aussi, au cinéma, de montage parallèle, "réunissant en des plans alternés deux événements distincts »). Le substantif, pour sa part, désigne une « comparaison suivie entre deux ou plusieurs sujets $^{1} »$ (quand on parle, par exemple, de faire ou d'établir un parallèle entre deux objets ou deux questions).

4 La « comparaison ", de son côté, avant de signifier un " rapport établi entre un objet et un autre terme, dans le langage» ( $d$ 'une manière parallèle à l'image ou à la métaphore ${ }^{2}$ ), indique «le fait d'envisager ensemble (deux ou plusieurs objets de pensée) pour en chercher les différences ou les ressemblances », ce qui implique l'idée de balance ou de parallèle, la capacité de « soutenir la comparaison » n'étant pas a priori donnée à tout le monde ou à n'importe quoi. Dans cette perspective, "sans 
comparaison " signifie "sans hésitation ", voire "très supérieur " ou "beaucoup mieux $^{3} »$ (mais, comme nous le verrons, les Anciens, plus mesurés et plus subtils encore, nous disent que cette assurance ne peut être définitive).

Dans ces conditions, il apparaît que les deux notions sont intrinsèquement liées. La comparaison procède par des mises en parallèle, tandis que le parallèle et l'analyse parallèle de la réalité impliquent une comparaison et un jugement. Or ces mécanismes de pensée étaient théorisés et enseignés dans les écoles de rhétorique de l'Antiquité.

\section{L'exercice rhétorique du parallèle}

6 Le "parallèle » (ou, comme le suggère Pierre Chiron, le " jugement parallèle ${ }^{4}$ ») est en effet l'un des exercices préparatoires (progymnasmata) que les adolescents du monde gréco-romain apprenaient à composer avec leur professeur de rhétorique ${ }^{5}$. Dans ce

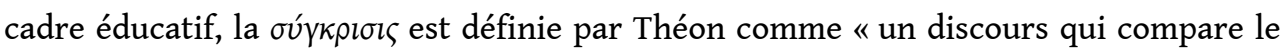
meilleur ou le pire» (trad. Patillon, p. 78), qu'il s'agisse de personnes (par exemple : Ajax et Ulysse) ou de choses (par exemple : la sagesse et le courage), et il est attendu de l'élève qu'il décrète une préférence ou prenne parti. Les qualités des objets examinés doivent être en rapport, dit Théon, qui ajoute : « Il faut préciser d'abord qu'on ne met pas en parallèle des éléments que sépare une grande différence (il est ridicule, en effet, celui qui se demande avec embarras si Achille est plus courageux ou si c'est Thersite), mais des éléments semblables et qui font l'objet d'un débat pour savoir lequel doit être mis avant l'autre, parce qu'on ne voit aucune supériorité de l'un sur l'autre » (Patillon p. 78, trad. modifiée). Ayant partie liée avec les exercices précédents de l'éloge et du blâme ${ }^{6}$, dont il utilise les techniques et les procédés, le parallèle ne concerne pas seulement des vertus ou des héros (comme ceux qui ont déjà été cités ou bien encore Achille et Hector, dont Aphthonios construit le parallèle en guise de modèle rédigé ${ }^{7}$ ), mais l'exercice peut aussi confronter des plantes, des activités, des temps, des lieux, des animaux ${ }^{8}$. S'agissant de la méthode à adopter pour mener le parallèle, Théon propose soit de développer, l'un à la suite de l'autre, deux discours, pour chaque objet, soit de mener la comparaison point par point (cette deuxième approche étant privilégiée par Aphthonios, qui condamne la première ${ }^{9}$ ). En définitive, comme le précise le PseudoHermogène, le jugement auquel l'exercice doit aboutir peut être variable, selon que l'on conclue à l'égalité des objets mis en regard ou bien que l'on établisse la supériorité de l'un soit en louant aussi l'autre, soit en le blâmant ${ }^{10}$. Dans tous les cas, la pratique pédagogique du parallèle est destinée à exercer "l'esprit de finesse ", "la capacité d'analyse ${ }^{11}$ ", bref l'esprit critique des étudiants, lesquels apprennent ainsi à innover dans leurs jugements et à refuser les préjugés.

\section{Comparaison n'est pas raison?}

7 Le parallèle, tel qu'il est défini par les pédagogues de l'Antiquité, est un exercice d'argumentation. Ainsi, en justifiant sa préférence pour le développement point contre point (plutôt qu'ensemble contre ensemble), Aphthonios affirme son refus de toute platitude et souligne la dimension agonistique (c'est-à-dire propre au débat) de l'énoncé1 ${ }^{12}$. Et quand il compare Achille à Hector, il le fait non seulement en sélectionnant un thème central (la bravoure) qui crée l'unité du propos, mais aussi en déclinant les topoi de l'éloge, à savoir: le lieu de naissance, l'origine et la lignée, 
l'éducation (ou la formation) et les actions, la mort. Dans les autres cas, les mêmes lieux de l'éloge de personne sont utilisés ou adaptés (par exemple, pour des activités, on parlera non de leur naissance, mais de ceux qui les ont initiées, de même que pour des plantes on évoquera les divinités qui les ont offertes à l'humanitéé ${ }^{13}$ ).

L'exercice s'avère ainsi exigeant, qui requiert, selon le Pseudo-Hermogène, vigueur, habileté, vivacité et rapidité de la part de l'orateur ${ }^{14}$. Par conséquent, les rhéteurs de l'Antiquité donnent tort à ceux qui considéreraient que «comparaison n'est pas raison ». Bien au contraire, par les comparaisons qu'il établit en recourant à la topique de l'éloge, le parallèle se construit comme une démonstration fondée sur des lieux qui constituent autant d'arguments destinés à prouver une préférence ou un jugement.

\section{Utilité et fortune du parallèle des Anciens}

Cet exercice, comme les autres de la série, constitue une préparation pour les futurs citoyens-orateurs de la vie réelle, car le "parallèle » inculque à la fois une technique rhétorique, un procédé littéraire et même un mécanisme de pensée.

D'abord, les auteurs de manuels prévoient eux-mêmes explicitement les réutilisations possibles de l'exercice du parallèle: dans l'éloge et le blâme, assurément, mais aussi dans la thesis et dans les discours délibératifs (où le parallèle s'avère précieux pour dégager ce qui est opportun et préférable dans une situation donnée), et dans les discours judiciaires (où l'on met en parallèle les crimes et les bonnes actions ${ }^{15}$ ). Utile à l'orateur, le parallèle constitue aussi, en tant que forme d'énoncé spécifique ou en tant que genre littéraire ou métalittéraire, un outil de choix pour le rhétoricien, pour l'historien, pour le poète et pour l'écrivain en général. En procédant par comparaison, Plutarque, par exemple, peut ainsi mettre en parallèle les grands hommes de la Grèce et du monde romain, tandis que d'autres auteurs confrontent de manière plus générale le présent au passé. Cette manière de raisonner a donc, pour les Anciens, des implications littéraires, culturelles, mais aussi historiques et politiques. Or la technique comparative du parallèle s'avère un mode de pensée important non seulement dans l'Antiquité, mais aussi dans la littérature postérieure. Devenu un procédé critique, le parallèle devient une manière d'appréhender le monde dont la fortune sera très riche (que l'on pense par exemple au Parallèle des Anciens et des Modernes que Charles Perrault publie en 1688).

11 Au-delà du domaine rhétorique et littéraire, il faut reconnaître, avec Pierre Chiron, que "l'opération de comparaison a une importance capitale dans toute vie intellectuelle ${ }^{16}$ ". Et comme Marcel Detienne, citant Bernardin de Saint-Pierre ( Il n'y a rien que l'esprit humain fasse si souvent que des comparaisons»), on ne peut que faire le constat suivant: " Pour le sens commun, comparer est un verbe bien éveillé. Il accompagne le regard du chaland, il anime l'œil du critique. Discerner, n'est-ce pas immédiatement établir des analogies, entrevoir des raisons, ébaucher des raisonnements ${ }^{17}$ ? » De fait, la pratique du parallèle s'observe jusque dans la vie quotidienne: en témoigne par exemple ce jeu des images que pratiquaient les banqueteurs de l'Antiquité et qui relève d'une « imagination comparative ${ }^{18}$ » propre à l'être humain. 


\section{Ne peut-on comparer que ce qui est comparable?}

12 Si Théon demande de ne pas comparer des sujets trop différents l'un de l'autre, comme Achille et Thersite, mais des éléments dont la supériorité fait débat, tous les rhéteurs ne partagent pas forcément cet avis. Ainsi Aphthonios conçoit l'exercice du parallèle d'une manière sensiblement différente : « Le parallèle, explique-t-il, est un discours qui compare au moyen d'une opposition et dont la conclusion met le plus du côté du comparé. Dans un parallèle il faut opposer le beau au bon, le mauvais au mauvais ou le bon au méchant, ou opposer le petit au plus grand. Bref, le parallèle est un éloge ou un blâme doubles, <ou un discours> composé d'un éloge et d'un blâme ${ }^{19}$ ». Ces propos démontrent qu'il n'y a pas de consensus (preuve en est que Théon se sent obligé de préciser sa préférence) et que la doctrine rhétorique des exercices préparatoires n'est pas restée figée. En d'autres termes, si certains considèrent qu'il ne faut comparer que ce qui est comparable, d'autres estiment que la confrontation d'éléments différents, voire opposés, permet aussi d'approfondir ses capacités d'analyse et d'argumentation. En ce sens, Plutarque choisit de comparer des Grecs et des Romains, quand bien même la culture grecque de son temps tend à ignorer totalement le fait romain. De même, comparer Achille à Thersite pourrait permettre, n'en déplaise à Théon, de jouer avec les opinions convenues, alors qu'un parallèle accordant sa préférence à Thersite s'apparenterait à un éloge paradoxal - même si un jugement inverse, plus probable, permet de réaffirmer, en les consolidant, des représentations en vigueur. Le parallèle est un jeu osé ou risqué ; et l'habileté de celui qui le pratique (tel Plutarque, donc, dans ses Vies parallèles) peut consister à dissimuler un message implicite : à charge pour l'auditeur ou le lecteur d'analyser lui-même le parallèle qui lui est proposé et d'en tirer un jugement définitif.

13 Comme l'explique Marcel Detienne, si l'on s'en tient à la maxime qui interdit de comparer ce qui ne serait pas comparable, le choix même des éléments à comparer implique aussi un jugement de valeur. Mais les Anciens suggèrent la "possibilité de construire ce qui peut être "comparable" ", car l'approche comparative fonctionne y compris pour ce qui n'est pas comparable a priori ${ }^{20}$. Il apparaît alors que les rhéteurs de l'Antiquité nous ont transmis non seulement un outil rhétorique, mais aussi une méthode d'analyse littéraire et philologique (qui implique de mettre en parallèle des textes ou des témoignages) et même une manière de pensée dont la valeur heuristique se vérifie par exemple dans le domaine de la littérature comparée ${ }^{21}$ ou dans celui de l'anthropologie. Créer ou découvrir des parallèles consiste à mettre en rapport des "relations immédiates» (ou moins évidentes), des «ensembles de relations ${ }^{22}$ », des convergences thématiques ou des perspectives qui ne se croisent pas, mais qui permettent d'approfondir le savoir. Établir des parallèles avec l'autre, l'ailleurs ou le passé procède d'un «comparatisme constructif ${ }^{23}$ ». Si l'exercice du parallèle est une expérimentation rhétorique, voire une manipulation qui ouvre des horizons, "le comparatiste expérimentateur se donne ainsi la liberté et le plaisir de démonter et de remonter des logiques partielles de pensée ${ }^{24}$ ».

En définitive, comparer, c'est-à-dire établir des parallèles, permet de rechercher des similitudes, de favoriser l'ouverture d'esprit, de "mettre en perspective des valeurs", de " porter un regard critique » sur soi (et pas seulement sur autrui), d'« apprendre à vivre avec les autres ${ }^{25} »$. Et il est fascinant de s'apercevoir que toutes ces possibilités et 
tous ces avantages sont au cœur de la démarche pédagogique des rhéteurs grecs et latins quand ils traitent du parallèle devant leurs élèves et avec eux.

\section{NOTES}

1. A. Rey, J. Rey-Debove, Le Petit Robert 1, Paris, Le Robert, 1986, p. 1354.

2. Sur ce sujet, voir par exemple (pour l'Antiquité) M. H. McCall, Ancient Rhetorical Theories of Simile and Comparison, Cambridge (Mass.), Harvard University Press, 1969.

3. A. Rey, J. Rey-Debove, op. cit., p. 347.

4. P. Chiron, Manuel de rhétorique. Comment faire de l'élève un citoyen, Paris, Les Belles Lettres, p. 159.

5. Sur la place de l'exercice du parallèle dans la liste et dans l'histoire des progymnasmata, cf. M. Patillon, Aelius Théon. Progymnasmata, Paris, CUF, 1997, p. LXXX-LXXXIII.

6. Du côté latin, l'exercice du parallèle, ou comparatio, découle aussi, selon Quintilien, Institution oratoire, II, 4, 21, de celui de l'éloge et du blâme ; la méthode en est analogue, mais le matériau dédoublé.

7. Aphthonios, Progymnasmata, X, 4-8.

8. Ibid., X, 2. Comparer Pseudo-Hermogène, Progymnasmata, VIII, 3-4. Quintilien, II, 4, 24, prévoit l'utilisation du parallèle dans l'exercice de la thesis, impliquant comparaison de deux éléments, y compris inanimés, comme dans les sujets d'école portant sur les mérites comparés de la vie rurale et de la vie urbaine, ou sur la supériorité du juriste sur le soldat.

9. Théon, 115, 7-12, p. 82 de l'éd. Patillon, op. cit. (où est cité le parallèle, établi dans le Banquet de Xénophon, entre l'amour de l'âme et celui du corps) ; Aphthonios, X, 3.

10. Ps.-Hermogène, VIII, 5.

11. P. Chiron, op. cit., p. 161-162.

12. Aphthonios, $X, 3$.

13. Ps.-Hermogène, VIII, 3-4.

14. Ibid., VIII, 6. Comparer Aphthonios, $X, 1$, qui insiste d'emblée sur l'habileté requise.

15. Théon, 60, 31 - 61, 6 (p. 3 de l'éd. Patillon). La comparatio est ainsi exploitée dans les états de cause, et notamment dans l'état selon le droit, ou status iuridicialis (Rhétorique à Hérennius II, 20-21) ; Cicéron, De l'invention I, 82, envisage de son côté le comparabile comme un moyen de réfuter une argumentation par une induction.

16. P. Chiron, op. cit., p. 160.

17. M. Detienne, Comparer l'incomparable, Paris, Seuil, 2000, p. 9.

18. Ibid., p. 20.

19. Aphthonios, X,1 (trad. Patillon modifiée, dans M. Patillon, Corpus rhetoricum. Anonyme: Préambule à la rhétorique. Aphthonios: Progymnasmata. En annexe Pseudo-Hermogène : Progymnasmata, Paris, CUF, 2008, p. 140-141).

20. M. Detienne, op. cit., p. 9.

21. Voir notamment J. Siess, " "Parallèle", un concept opératoire en littérature comparée? ", Revue de littérature comparée, 298, 2001, p. 225-230.

22. M. Detienne, op. cit., p. 10.

23. Ibid.., p. 11, 13, 20.

24. Ibid.., p. 15. 
25. Ibid.., p. 56, 59.

\section{AUTEURS}

JOHANN GOEKEN

Université de Strasbourg (Centre d'Analyse des Rhétoriques Religieuses de l'Antiquité)

\section{CATHERINE SCHNEIDER}

Université de Strasbourg (Centre d'Analyse des Rhétoriques Religieuses de l'Antiquité) 